\title{
Imaging Specific Protein Labels on Eukaryotic Cells in Liquid with Scanning Transmission Electron Microscopy
}

\author{
N. de Jonge, ${ }^{* * * *}$ M.J. Dukes, ${ }^{*}$ E. A. Ring, ${ }^{*}$ D. Drouin, ${ }^{* * *}$ and D.B. Peckys*****
}

*Molecular Physiology and Biophysics, Vanderbilt University Medical Center, 2215 Garland Ave, Nashville TN 37232-0615

**Materials Science and Technology Division, Oak Ridge National Laboratory, 1 Bethel Valley Rd., Oak Ridge, TN 37831-6064

***Université de Sherbrooke, Electrical and Computer Engineering, Sherbrooke, 2500 boul. Université, Quebec, J1K 2R1, Canada

****Center for Environmental Biotechnology, University of Tennessee, Dabney Hall, Knoxville, Tennessee, TN 37996-1605

A central scientific challenge is to understand the structure and dynamics of protein complexes underlying cellular function. Biochemical techniques used to identify such complexes would be enhanced by protein-scale resolution (on the order of a few nanometers) imaging of specific molecular positions in the context of intact cells. Currently, though, nanometer resolution can only be achieved at the cost of less direct imaging of the unperturbed cell. Cellular ultrastructure is traditionally studied by transmission electron microscopy (TEM), which yields nanometer resolution on embedded and stained sections, or cryo sections. These cells are neither intact nor in their native liquid state. Light microscopy is used to image protein distributions via fluorescent labels on cells in liquid to investigate cellular function, but even recent improvements in resolution by "nanoscopy" techniques [1] are still insufficient to resolve the constituents of protein complexes. Thus, development of techniques capable of high-resolution imaging in native cellular states would contribute significantly to our understanding of cellular function at a molecular level. Two technological achievements from the last decade, the introduction of nanoparticles as specific protein labels [2] and the development of silicon nitride membranes for use as electron-transparent windows in a liquid compartment [3], have led to the introduction of a novel concept to achieve nanometer resolution on tagged proteins in cells $[4,5]$. Eukaryotic cells in liquid are placed in a micro-fluidic chamber with a thickness of up to $10 \mu \mathrm{m}$ contained between two ultra-thin and electron-transparent windows, as in Figure 1. The specimen is then imaged with the scanning transmission electron microscope (STEM). On account of the atomic number (Z) contrast of the STEM, nanoparticles of a high-Z material, such as gold, can be detected within the background signal produced by a low-Z liquid, such as water. The nanoparticles specifically attached to proteins can then be used to study protein distributions in whole cells in liquid, similar as proteins tagged with fluorescent labels can be used to study protein distributions in cells with fluorescence microscopy.

A spatial resolution of $1.5 \mathrm{~nm}$ was achieved on gold nanoparticles placed above a water layer of a thickness of $4 \mu \mathrm{m}$, consistent with theoretical predictions, and with Monte Carlo simulations of the STEM imaging. The resolution decreased for nanoparticles placed deeper in the liquid due to beam broadening. COS7 fibroblast cells were labeled with gold nanoparticles conjugated with epidermal growth factor (EGF). Intact fixed cells in liquid were imaged with STEM with a spatial resolution of $4 \mathrm{~nm}$, while providing imaging at $20 \mu$ s pixel dwell time. The high spatial resolution of liquid STEM obtained on sample volumes compatible with whole eukaryotic cells is not achievable with a liquid cell for TEM imaging, because the TEM contrast mechanism limits high-resolution imaging 
of low-Z materials to a thickness of about $0.5 \mu \mathrm{m}$. In the case of thin and weakly scattering samples, TEM yields a better resolution than STEM, but in our case, STEM offers a particular advantage based on the imaging of high-Z labels. The resolution we have achieved also surpasses alternative approaches, such as the imaging of back-scattered-, or transmitted electrons with a scanning electron microscope (SEM), or X-ray microscopy. For fixed samples, liquid STEM presents a novel alternative to fluorescence microscopy, but with a factor of 50 better resolution. The use of fluorescent nanoparticles, such as quantum dots (QDs), allows liquid STEM images to be correlated with fluorescence images, such that the biological state of a cell can first be determined via light microscopy. Eukaryotic cells were grown directly on microchips for the micro-fluidics chamber, fixed, and imaged with fluorescence microscopy. The micro-fluidics chamber was then assembled to image the intact cells in liquid with STEM. Correlation of the obtained high-resolution images, showing individual QDs, with the cellular regions, as imaged with fluorescence microscopy, was accomplished. None of the existing correlative light- and electron microscopy techniques are capable of imaging whole cells in liquid, and require sample preparation into plastic thin sections, or cryo sections. Thus, liquid STEM presents an innovative approach for the imaging of single molecules in whole cells, which is significantly improved over existing methods in spatial resolution and imaging speed. Liquid STEM has the potential to provide the combination of light microscopy functionality and electron microscopy resolution. [6]

\section{References}

[1] S.W. Hell, Science 316 (2007) 1153.

[2] Y. Xiao et al., Science 299 (2003) 1877.

[3] M.J. Williamson et al., Nature Materials 2 (2003) 532.

[4] N. de Jonge et al., Proc. Natl. Acad. Sci. 106 (2009) 2159.

[5] D.B. Peckys et al., PLoS One 4 (2009) e8214.

[6] We are grateful to D.W. Piston, S. Head, T.E. McKnight, and Protochips Inc.. A Portion of this research was conducted at the SHaRE User Facility, which is sponsored by the Division of Scientific User Facilities, Office of Basic Energy Sciences, U.S. Department of Energy. Research supported by Vanderbilt University Medical Center, NIH grant R01GM081801 (NJ), and NIH grant R01-RR018470 (to P. Mazur for DBP).

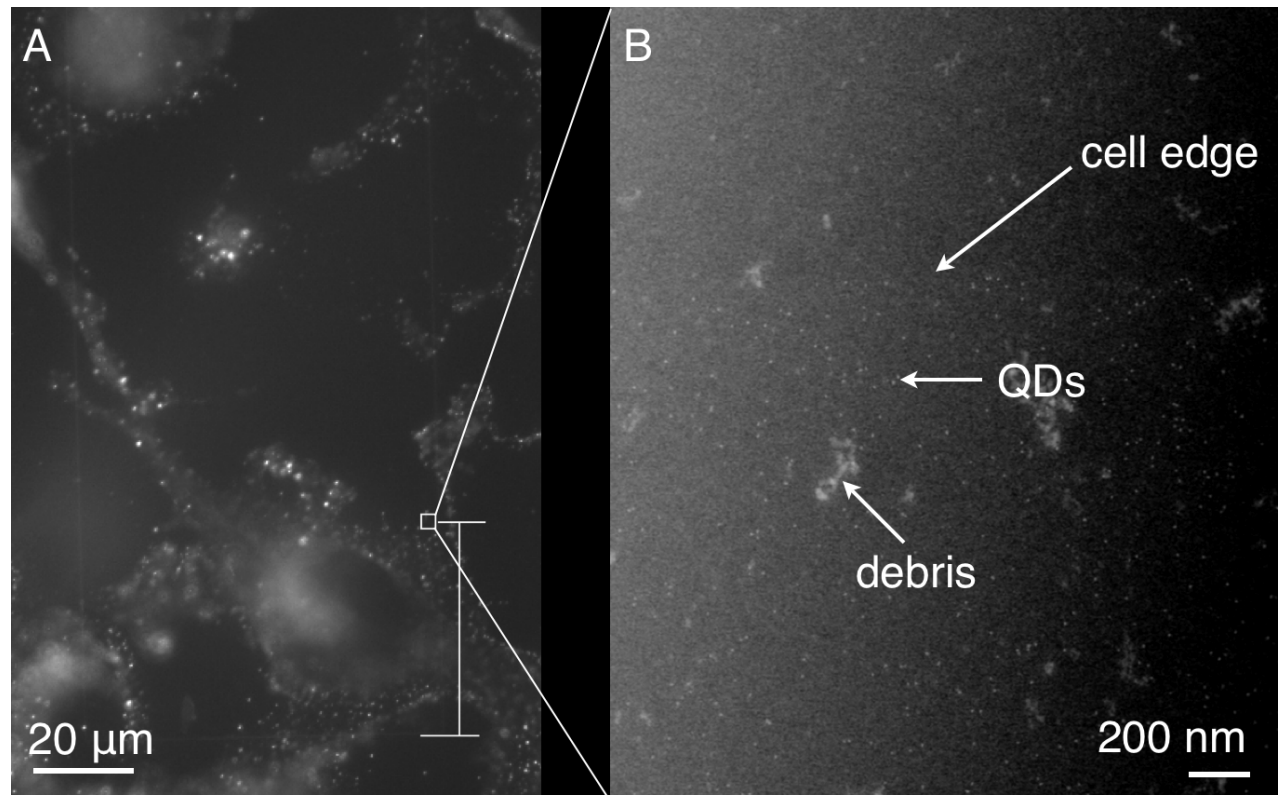

FIG. 1. Correlative fluorescence microscopy and liquid STEM of intact fixed eukaryotic cells in saline water. (A) Fluorescence image of microchip with COS7 cells showing the regions with EGF-QD labeling. (B) Liquid STEM image of region indicated with square in (A). Individual QDs and the edge of the cell can be discerned. Some debris is also visible. 